\title{
ESTUDO DE PROPRIEDADES GRANULOMÉTRICAS DOS AGREGADOS DE RESÍDUOS DE CONCRETO DESTINADOS À APLICAÇÕES EM NOVOS MATERIAIS
}

Jacqueline Roberta Tamashiro ${ }^{1,2 *}$; Vitória Domingues Galante ${ }^{1,3}$; Patricia Alexandra Antunes ${ }^{1}$; Rebeca Delatore Simões ${ }^{1,4}$

\footnotetext{
${ }^{1}$ Universidade do Oeste Paulista - UNOESTE, ${ }^{2}$ Mestrado em Meio Ambiente e Desenvolvimento Regional, ${ }^{3}$ Graduação em Engenhara Civil, Presidente Prudente, SP.

${ }^{4}$ Universidade Estadual Paulista, Campus de Tupã, SP. E-mail: arquiteta.jtamashiro@hotmail.com.

Agência de fomento: CAPES
}

\section{RESUMO}

A reutilização dos resíduos de construção para aplicação em novos materiais vem sendo uma alternativa sustentável de reinserção no ciclo construtivo. O CONAMA já regulamenta a reutilização de componentes de concreto como agregados. No entanto, a variabilidade destes resíduos é um fator preponderante nas dosagens, que pode resultar em alterações na qualidade final. O objetivo deste trabalho é avaliar resíduos de concreto e areia da região administrativa de Presidente Prudente, SP, por meio de comparação de propriedades granulométricas para aplicações em novos materiais. O material analisado foi homogeneizado, quarteado, limpo, seco, pesado, peneirado, e, separado em quantidades retidas e acumuladas. Ao analisar a distribuição gráfica das curvas granulométricas foi possível constatar que tanto a areia, como o resíduo de concreto atendem aos parâmetros de utilização requeridos pela ABNT às NBR 7217 (1987), NBR NM 248 (2003), NBR 7211 (2005) e NBR 15116 (2004) de agregados e granulometria.

Palavras-chave: granulometria; agregados; resíduos de concreto; areia natural.

\section{STUDY OF GRANULOMETRIC PROPERTIES OF WASTE CONCRETE AGGREGATES INTENDED FOR APPLICATIONS IN NEW MATERIALS}

\begin{abstract}
The reuse of construction waste for application in new materials has been a sustainable alternative to reinsertion in the construction cycle. The CONAMA already regulates the reuse of concrete components as aggregates. However, the variability of these residues is a preponderant factor in the dosages, which may result in changes in the final quality. The objective of this work is to evaluate concrete and sand residues of the administrative region of Presidente Prudente, SP, by comparing granulometric properties for applications in new materials. The analyzed material was homogenized, quarteted, cleaned, dried, weighed, sifted, and separated in retained and accumulated amounts. When analyzing the graphical distribution of the grain size curves, it was possible to verify that both the sand and the concrete residue meet the parameters of use required by the ABNT NBR 7217 (1987), NBR NM 248 (2003), NBR 7211 (2005) and NBR 15116 (2004) of aggregates and granulometry.
\end{abstract}

Keywords: granulometry; aggregates; concrete waste; natural sand. 


\section{INTRODUÇÃO}

Atualmente, diversos avanços tecnológicos de reciclagem e reaproveitamento de resíduos podem ser vistos no contexto da construção civil. Segundo a ABRELPE (2015), no Brasil cerca de $60 \%$ do volume do resíduo sólido urbano vêm da construção civil e até $70 \%$ deste volume pode ser reutilizado. Ainda, os dados do Panorama dos Resíduos Sólidos no Brasil, ABRELPE (2015), mostraram que os municípios brasileiros coletaram aproximadamente 44 milhões de toneladas de Resíduos da Construção Civil (RCC). Para Marques, Oliveira e Picanço (2013), os materiais mais presentes nos resíduos de construção são a argamassa e o concreto, responsáveis por $90 \%$ da composição média dos materiais. Segundo Menezes (2011), as formas nas quais o resíduo de concreto é empregado no Brasil é como componente agregado em bases e sub-bases de pavimentação. Também é utilizado como concreto asfáltico pré-misturado a frio misturado ao ligante asfáltico. Hu, Souza e Genarini (2017), Campos \& Paulon (2015), Thomas et al. (2013) e Gonçalves (2011), afirmam que os resíduos podem ser reutilizados como agregados também na produção de concretos. No entanto, para sua substituição é fundamental que a granulometria dos Agregados de Resíduos de Concreto (ARC) sejam compatíveis com os agregados miúdos naturais utilizados tradicionalmente na construção civil.

Este trabalho teve como objetivo avaliar a viabilidade do uso de concreto e a areia natural (agregado convencional) da região administrativa de Presidente Prudente, SP, e o concreto reciclado como agregado miúdo, por meio de um estudo comparativo das propriedades granulométricas. Verificar se os resultados obtidos atendem as normas da ABNT para agregados determinação da composição granulométrica, NBR 7217 (1987), NBR NM 248 (2003); agregados para concreto, NBR 7211 (2005) e agregados reciclados de resíduos sólidos da construção civil utilização em pavimentação e preparo de concreto sem função estrutural - requisitos, NBR 15116 (2004).

\section{METODOLOGIA MATERIAIS}

A areia natural fora cedida pelo Laboratório de Engenharia Civil da Universidade do Oeste Paulista (UNOESTE), com origem de extração em Presidente Epitácio, SP. O método de coleta e amostragem foi em pilhas em atendimento as normas da ABNT NBR NM 26 (2009) de amostragem de agregados e ABNT NBR NM 27 (2001) de redução da amostra de campo para ensaios de laboratório.

A amostra de resíduo de concreto foi selecionada, por ser de caráter científico, para não haver possíveis agentes externos (como solventes, óleos e graxas, vernizes, tintas, ou até mesmo a presença de aditivos químicos) que pudessem vir a comprometer os resultados. As unidades experimentais, que compuseram a amostra de resíduos de concreto foram coletadas dentro do município de Presidente Prudente, em dias alternados e em quantidades aproximadas de 100 a $120 \mathrm{~kg}$ (ABNT NBR 10007, 2004). A composição da amostra de estudo se deu pela mistura das unidades experimentais pela técnica de amostras parciais utilizando o processo de quarteamento, alcançando assim a quantidade mínima exigida pela NBR NM 27 (2001). Posteriormente, a amostra passou pelo processo de britagem num moinho de martelos com potência de $5 \mathrm{hp}$, no Laboratório de Materiais Cerâmicos da UNESP (LAMAC).

Respeitando-se as normas para agregados reciclados, foram utilizadas respectivamente: ABNT NBR 15116 (2004) de agregados reciclados de resíduos sólidos da construção civil utilização em pavimentação e preparo de concreto sem função estrutural - Requisitos; ABNT NBR NM 27 (2001) de redução da amostra de campo para ensaios de laboratório e Resolução de no 307 pelo (CONAMA, 2002). 


\section{COMPOSIÇÃO GRANULOMÉTRICA}

Os ensaios de granulometria da areia natural ou agregado miúdo, foram executados de acordo com os parâmetros da ABNT NBR 9935 (2011) de terminologia de agregados; ABNT NBR 7211 (2005) de especificação para agregados de concreto; ABNT NBR 7217 (1987) de agregados determinação da composição granulométrica; ABNT NBR NM ISO 3310-1 (2010) de peneiras de ensaio - requisitos técnicos e verificação.

A aparelhagem e instrumentos auxiliares utilizados para a realização do ensaio foram uma balança de precisão; estufa térmica; peneiras das séries normal e intermediária da marca Granutest ${ }^{\circledR}$ e Solotest ${ }^{\circledR}$, agitador mecânico de peneiras, bandejas metálicas, fundo avulso de peneiras e pincéis (ABNT NBR 7217, 1987).

De acordo com a ABNT NBR 7217 (1987) as amostras de areia foram secas em estufa em temperatura entre 105 a $110^{\circ} \mathrm{C}$. Depois de estabilizar à temperatura ambiente, foi determinada sua massa dividida em duas partes de $500 \mathrm{~g}$; totalizando em $1 \mathrm{~kg}$ de agregado miúdo a cada ensaio realizado. $O$ processo de agitação mecânica do conjunto de peneiras foi realizado em 5 minutos. Ao determinar a massa total do material retido em cada peneira, a somatória das massas não poderia diferir em mais que $0,3 \%$ da massa seca da amostra, inicialmente introduzida no conjunto de peneiras (ABNT NBR 7217, (1987).

\section{RESULTADOS}

Os dados obtidos são apresentados na Tabela 1. Observa-se, que os grãos que ficaram retidos nas peneiras de abertura de malha $\geq 12,5 \mathrm{~mm}$ correspondem a média de $2,49 \%$ do total analisado. No entanto, em maior quantidade foram os grãos passantes na peneira com abertura de malha de $9,5 \mathrm{~mm}$ e ficaram retidos no fundo, ou seja, passantes à peneira de $150 \mu \mathrm{m}$ e retidos no prato. Estes agregados, por sua vez, são denominados como agregados miúdos conforme a ABNT NM 52 (2003). Os ensaios apontaram que os agregados miúdos de areia natural representam em sua maioria $97,61 \%$ da massa total ensaiada, enquanto os grãos de ARC representam $97,41 \%$ da massa total correspondente à $1000 \mathrm{~g}$. Portanto, os valores dos dados granulométricos de ARC são compatíveis com os de areia natural, onde diferem em apenas 0,20\% da massa retida entre $9,5 \mathrm{~mm}$ a $150 \mu \mathrm{m}$ (areia/ARC). 
Tabela 1. Composição granulométrica de resíduos de concreto e areia

\begin{tabular}{|c|c|c|c|c|c|c|c|}
\hline \multirow{2}{*}{\multicolumn{2}{|c|}{$\begin{array}{c}\text { PENEIRAS } \\
\text { Peneira com abertura de } \\
\text { malha (ABNT NBR NM ISO } \\
\text { 3310-1) }\end{array}$}} & \multirow{3}{*}{ Peso (g) } & \multicolumn{2}{|c|}{$\begin{array}{c}\text { AREIA } \\
\text { \% em massa, retida } \\
\text { acumulada }\end{array}$} & \multirow[t]{3}{*}{$\begin{array}{r}\text { RE } \\
\text { Peso (g) }\end{array}$} & \multicolumn{2}{|c|}{$\begin{array}{l}\text { SÍDUO DE CONCRETO } \\
\text { \% em massa, retida } \\
\text { acumulada }\end{array}$} \\
\hline & & & \multirow{2}{*}{$\%$ Retida } & \multirow{2}{*}{$\%$ Acum. } & & \multirow{2}{*}{$\%$ Retida } & \multirow{2}{*}{$\%$ Acum. } \\
\hline Normal & Intermediária & & & & & & \\
\hline $75 \mathrm{~mm}$ & - & 0 & $0 \%$ & $0 \%$ & 0 & $0 \%$ & $0 \%$ \\
\hline- & $63 \mathrm{~mm}$ & 0 & $0 \%$ & $0 \%$ & 0 & $0 \%$ & $0 \%$ \\
\hline- & $50 \mathrm{~mm}$ & 0 & $0 \%$ & $0 \%$ & 0 & $0 \%$ & $0 \%$ \\
\hline $37,5 \mathrm{~mm}$ & - & 0 & $0 \%$ & $0 \%$ & 0 & $0 \%$ & $0 \%$ \\
\hline- & $31,5 \mathrm{~mm}$ & 0 & $0 \%$ & $0 \%$ & 0 & $0 \%$ & $0 \%$ \\
\hline- & $25 \mathrm{~mm}$ & 0 & $0 \%$ & $0 \%$ & 0 & $0 \%$ & $0 \%$ \\
\hline $19 \mathrm{~mm}$ & - & 0 & $0 \%$ & $0 \%$ & 0 & $0 \%$ & $0 \%$ \\
\hline- & $12,5 \mathrm{~mm}$ & 23,95 & $2,40 \%$ & $2,40 \%$ & 25,91 & $2,59 \%$ & $2,59 \%$ \\
\hline $9,5 \mathrm{~mm}$ & - & 0,00 & $0,00 \%$ & $2,40 \%$ & 0 & $0,00 \%$ & $0,00 \%$ \\
\hline- & $6,3 \mathrm{~mm}$ & 0,00 & $0,00 \%$ & $2,40 \%$ & 0 & $0,00 \%$ & $0,00 \%$ \\
\hline $4,75 \mathrm{~mm}$ & - & 3,07 & $0,31 \%$ & $2,70 \%$ & 8,63 & $0,86 \%$ & $3,45 \%$ \\
\hline $2,36 \mathrm{~mm}$ & - & 21,71 & $2,17 \%$ & $4,87 \%$ & 22,80 & $2,28 \%$ & $5,73 \%$ \\
\hline $1,18 \mathrm{~mm}$ & - & 69,40 & $6,94 \%$ & $11,81 \%$ & 118,26 & $11,83 \%$ & $17,56 \%$ \\
\hline $600 \mu \mathrm{m}$ & - & 271,53 & $27,15 \%$ & $38,97 \%$ & 180,17 & $18,02 \%$ & $35,58 \%$ \\
\hline $300 \mu \mathrm{m}$ & - & 400,96 & $40,10 \%$ & $79,06 \%$ & 348,15 & $34,82 \%$ & $70,39 \%$ \\
\hline $150 \mu \mathrm{m}$ & - & 175,47 & $17,55 \%$ & $96,61 \%$ & 240,57 & $24,06 \%$ & $94,45 \%$ \\
\hline \multicolumn{2}{|r|}{ resíduo } & 33,91 & $3,39 \%$ & $100 \%$ & 55,51 & $5,55 \%$ & $100 \%$ \\
\hline \multicolumn{2}{|r|}{ TOTAL } & 1000 & $100 \%$ & & 1000 & $100 \%$ & \\
\hline
\end{tabular}

Fonte: (Dos autores, 2017)

Assim, no Gráfico 1 são comparadas as curvas granulométricas do resíduo de concreto e da areia natural. 
Gráfico 1. Distribuição granulométrica de agregados miúdos

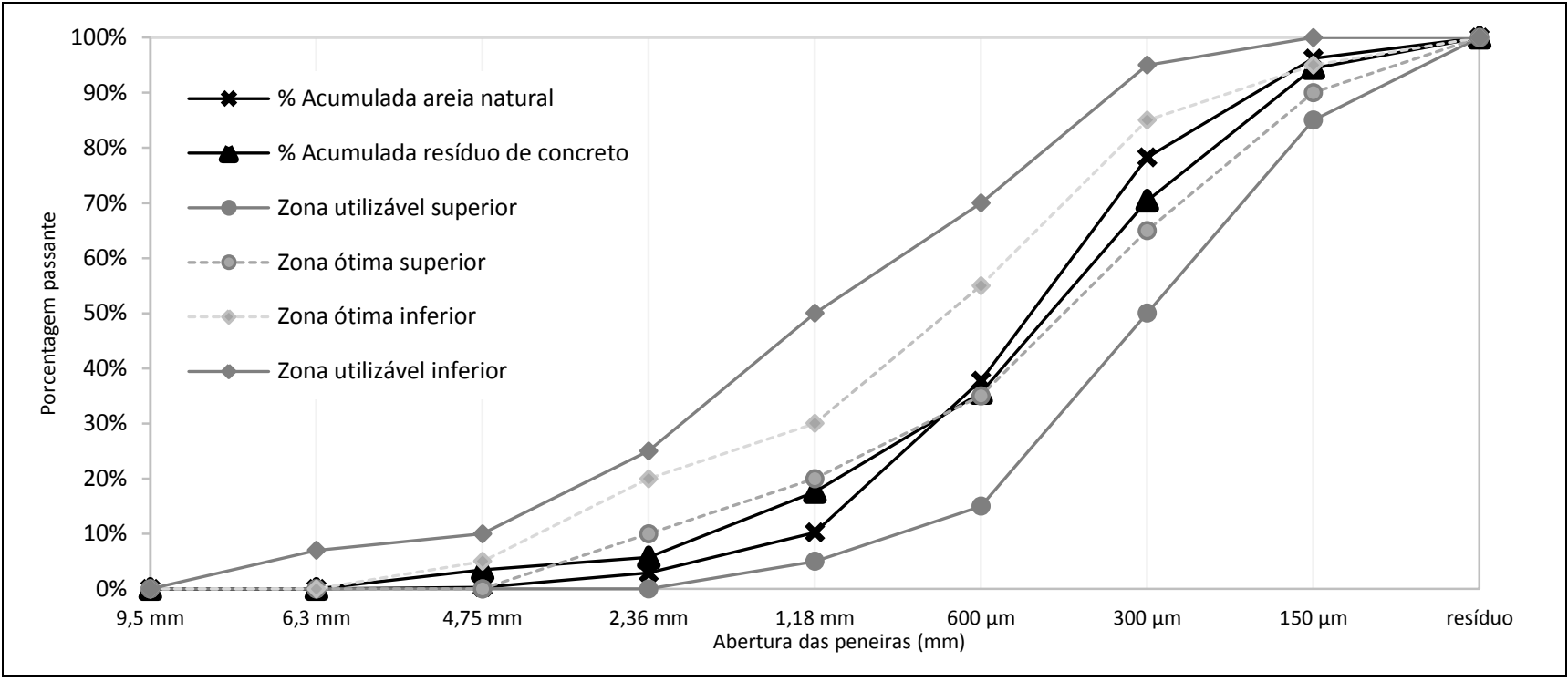

Fonte: (Dos autores, 2017)

A distribuição da granulometria mostra que a areia natural apresenta tendência e se encaixa na "zona utilizável superior" a aceitável pela ABNT NBR 248 (2003). A partir do módulo de finura correspondente à $600 \mu \mathrm{m}$ a curva se encontra nos parâmetros exigidos entre a "zona ótima inferior" e "ótima superior". Observou-se que nas peneiras de 9,5mm até aproximadamente $600 \mu \mathrm{m}$, a areia natural acumulada mostrou tendência à "zona utilizável superior" para grãos maiores, o que descarta a possibilidade de serem grãos mais finos ou pulverulentos mediante às peneiras de 300 e $150 \mu \mathrm{m}$.

Os resultados das medidas de granulometria dos agregados miúdos de areia e dos ARC, apresentam semelhanças granulométricas. Nota-se principalmente os valores aproximados nos pontos de intersecção na "zona ótima superior" entre as peneiras de 600 e $150 \mu \mathrm{m}$, correspondentes à 38,97\% (areia) e 35,58\% (ARC).

\section{DISCUSSÃO}

Segundo Neville (2016) a grande concentração de material pulverulento como, siltes e pós finos, prejudicam no processo de hidratação do cimento, pois devido a sua finura e grande área superficial necessitam de maior quantidade de água para compensação de todas as partículas, aumentando consecutivamente o fator água/cimento e diminuindo a resistência do concreto.

A critério do módulo de finura do ARC, o mesmo mostrou-se mais fino em granulometria do que a areia natural. Entre as peneiras de 6,3mm a 4,75mm do Gráfico 1 a curva estabelece que os agregados constituídos por resíduos estão na zona utilizável inferior e ótima inferior. De acordo com Tenório (2007), Angulo (2000), Leite (2001) o teor de finos, ou de granulometria inferior a $0,15 \mathrm{~mm}$ e pulverulentos dos agregados reciclados é bastante variável, pois depende da composição do resíduo de origem e do equipamento usado na fragmentação. Em estudos, Tenório (2007) afirma que $60 \%$ de finos em massa pode ser encontrada nestes resíduos, porém "agregados de concreto reciclado de diferentes qualidades não apresentam variações significativas na granulometria e podem apresentar curvas parecidas com a de agregados naturais, quando retiradas as parcelas finas" (TENÓRIO, 2007, p.36). Porém, Neville (2016) admite que deve ser considerada a tipologia do agregado, pois em agregados artificiais existe a presença de materiais cimentícios ou filler, que explicaria a porcentagem de materiais ultrafinos $\geq 125 \mu \mathrm{m}$ ou resíduo de fundo no Gráfico 1. 
Neville (2016) ainda afirma que não existe uma curva granulométrica ideal. Sugere-se que os principais fatores que controlam a granulometria dos agregados são: a área superficial do agregado, que determina a quantidade de água necessária a molhagem dos sólidos; o volume relativo ocupado por cada agregado; a trabalhabilidade da pasta e a tendência a segregação.

É recomendável que a mistura do concreto seja coesa e trabalhável, com quantidade de material suficiente menor que $300 \mu \mathrm{m}$. Ainda observa que partículas de $<300 \mu \mathrm{m}$ devem compor a dosagem, para que não resulte em um concreto com excesso de partículas "ásperas", ou seja, em uma só dimensão (graúdas ou miúdas somente). Quando uma mistura é áspera, a distribuição granulométrica apresenta um salto abrupto no meio da curva. Diante deste fato é necessária uma quantidade adequada de materiais finos, estabelecidos em teores mínimos de partículas passantes na peneira de $300 \mu \mathrm{m}$ e, em alguns casos, também na peneira de $150 \mu \mathrm{m}$ (NEVILLE, 2016).

\section{CONCLUSÃO}

O agregado composto por resíduo de concreto apresentou características granulométricas semelhantes aos agregados miúdos naturais, no caso a areia. Ao analisar a distribuição gráfica das curvas granulométricas, foi possível constatar que o resíduo de concreto se encontra nos parâmetros de utilização requeridos pelas normas da Associação Brasileira de Normas Técnicas (ABNT).

Recomenda-se que sejam realizadas novas análises a respeito do formato dos grãos. Visto que, são produtos resultantes de ações mecânicas, a tipologia dos grãos pode variar de acordo com os britadores utilizados.

Contudo, o uso de agregados artificiais ou ARC, mostraram através dos resultados que podem ser utilizados em substituição ou reutilização em novas composições de materiais de construção. Contanto que sejam ajustadas através de dosagens específicas para cada uso e aplicação.

\section{AGRADECIMENTOS}

A Coordenação de Aperfeiçoamento de Pessoal de Nível Superior (CAPES) pelo subsídio aos estudos.

Ao Programa de Pós-Graduação de Mestrado em Meio Ambiente e Desenvolvimento Regional (MMADRE).

Aos laboratórios de Materiais Cerâmicos da UNESP (LAMAC) e de Engenharia Civil da Universidade do Oeste Paulista (UNOESTE).

\section{REFERÊNCIAS}

ANGULO, S.C. Variabilidade de agregados graúdos de resíduos de construção e demolição reciclados. São Paulo, 2000. 155p. Dissertação (Mestrado) - Escola Politécnica, Universidade de São Paulo, https://doi.org/10.11606/D.3.2000.tde-05102005-112833.

ASSOCIAÇÃO BRASILEIRA DA LIMPEZA PÚBLICA E RESÍDUOS ESPECIAIS, ABRELPE. Panorama de Edificações. 2015. Disponível em: <http://www.abrelpe.org.br/panorama_edicoes.cfm> Acesso em: 04 ago. 2017.

ASSOCIAÇÃO BRASILEIRA DE NORMAS TÉCNICAS, ABNT. Agregados- determinação da composição granulométrica: NBR 7217. Rio de Janeiro, 1987.

ABNT. Agregados- determinação da composição granulométrica: NBR NM 248. Rio de Janeiro, 2003. 
ABNT. Agregados miúdos - Determinação da massa específica e massa específica aparente: NBR NM 52. Rio de Janeiro, 2003.

, ABNT. Agregados para concreto: NBR 7211. Rio de Janeiro, 2005.

ABNT. Agregados reciclados de resíduos sólidos da construção civil - utilização em pavimentação e preparo de concreto sem função estrutural - requisitos: NBR 15116. Rio de Janeiro, 2004.

, ABNT. Amostragem de agregados: NBR NM 26. Rio de Janeiro, 2009.

ABNT. Amostragem de resíduos sólidos: NBR 10007. Rio de Janeiro, 2004.

, ABNT. Peneiras de ensaio - Requisitos técnicos e verificação - Parte 1: Peneiras de ensaio com tela de tecido metálico: NBR NM ISO 3310-1. Rio de Janeiro, 2010.

ABNT. Redução da amostra de campo para ensaios de laboratório: NBR NM 27. Rio de Janeiro, 2001.

, ABNT. Terminologia de agregados: NBR 9935. Rio de Janeiro, 2011.

CAMPOS, M. A.; PAULON, V. A. Utilização de agregados alternativos de isoladores elétricos de porcelana em concretos. Concreto y cemento. Investigación y desarrollo, v.7, n.1, p. 30-43, 2015.

Conselho Nacional Do Meio Ambiente, CONAMA. Resolução n. 307 de 5 julho de 2002. Publicada no DOU n. 136: http://www.mma.gov.br/port/conama/legiabre.cfm?codlegi=307. Acesso em: 10 mai. 2016.

GONÇALVES, M. S. Análise da viabilidade técnica de utilização de resíduos de concreto oriundos da pré-fabricação como agregado graúdo para a produção de novos concretos. 2011. $116 \mathrm{f}$. Dissertação (Mestrado) - Programa de Pós-graduação em Engenharia Civil, Universidade do Vale do Rio dos Sinos, UNISINOS, São Leopoldo, 2011.

HU, J.; LEVI SOUZA, I.; CORTÊS GENARINI, F. Engineering and environmental performance of ecoefficient self-consolidating concrete (Eco-SCC) with low powder content and recycled concrete aggregate. Journal of Sustainable Cement-Based Materials, v.6, n.1, p.2-16, 2017, https://doi.org/10.1080/21650373.2016.1230901.

LEITE, M.B. Avaliação de propriedades mecânicas de concretos produzidos com agregados reciclados de resíduos de construção e demolição. 2001. 270f. Tese (Doutorado) - Programa de Pós-Graduação em Engenharia Civil, Universidade Federal do Rio Grande do Sul, Porto Alegre, 2001.

MARQUES, O.B.; OLIVEIRA, R.M.S.; PICANÇO, A.P. Resíduos de construção civil: geração e alternativas para reciclagem em um canteiro de obras de pequeno porte. Rev. Engenharia Ambiental - Espírito Santo do Pinhal, v. 10, n. 2, p. 143-156, mar./abr. 2013.

MENEZES, M.S. et al. Panorama dos Resíduos de Construção e Demolição. RQI - Revista de Química Industrial, Rio de Janeiro, RJ, v. 79, n. 733, p.17-21, out. 2011. 
NEVILLE, A M. Propriedades do Concreto. 5. ed. Porto Alegre: Bookman, 2016. 888 p.

TENÓRIO, J. J. L. Avaliação de propriedades do concreto produzido com agregados reciclados de resíduos de construção e demolição visando aplicações estruturais. 2007. 138f. Tese (Doutorado) Programa de Pós-graduação em Engenharia Civil da Universidade Federal de Alagoas, Universidade Federal de Alagoas, Maceió, 2007.

THOMAS, C. et al. Durability of recycled aggregate concrete. Construction and Building Materials, v.40, p.1054-1065, 2013, https://doi.org/10.1016/i.conbuildmat.2012.11.106. 